\title{
LAS NUEVAS TECNOLOGÍAS DE INFORMACIÓN Y COMUNICACIÓN EN LOS PROCESOS DE ENSEÑANZA Y APRENDIZAJE: REPRESENTACIÓN DEL CONOCIMIENTO Y EDUCACION VIRTUAL
}

\author{
Mariela Rivero Carrillo* \\ Herminia Quiceno Valencia**
}

\begin{abstract}
This article aims at introducing the research group in educational informatics existing at the Autonoma University of Manizales -UAM- (Colombia). First, a brief description of the group is presented and some of the events in which it has participated are mentioned, such as the proposal known as Ludomatica, which has been implemented in some primary schools in the state of Caldas, Colombia. Then, the mission, objectives and theoretical, methodologícal and technological accomplishments of the group are defined. With reference to its theoretical accomplishments, some new conceptualizations have been consolidated about the meaning of Educational Informatics andabout the role of the New information and Communications Technologies in education. Concerning the methodological advances, some teaching processes in some virtual courses at UAM were characterized from the perspective of a Master's degree thesis project. From the technological point of view it is worth highlighting the effort with reference to developing educational software on the basis of thesis projects at the School of Telecommunications and Systems Engineering at UAM.
\end{abstract}

\section{RESUMEN}

Este artículo presenta el grupo de investigación en informática educativa de la Universidad Autónoma de Manizales (UAM), en primer lugar se hace una corta reseña del grupo mencionando algunas de las actividades en las que se ha participado, como por ejemplo la apropiación de la propuesta Ludomática en algunas escuelas de Caldas.

Además se presenta la misión del grupo, sus objetivos y avances en lo teórico, metodológico y tecnológico. Con respecto a los avances en lo teórico, se han realizado algunas conceptualizaciones alrededor del significado de informática educativa y el papel de las nuevas tecnologías de información y comunicaciones en la educación. En lo metodológico se realizó el año anterior un trabajo de grado a nivel de Maestría en el cual se caracterizaron los procesos de enseñanza en algunos cursos virtuales de la UAM y en lo tecnológico se menciona el trabajo que se hace con respecto al desarrollo de software

\footnotetext{
* mrivero(ómanizales.autonoma.edu.co. Docente Facultad de Ingeniería de Sistemas y

Telecomunicaciones. Universidad Autónoma de Manizales.

** quiher@manizales.autonoma.edu.co. Docente Facultad de Ingeniería de Sistemas y Telecomunicaciones. Universidad Autónoma de Manizales.
} 
educativo desde los proyecto de grado de la Facultad de Ingeniería de Sistemas y Telecomunicaciones de la UAM.

Palabras clave: Multimedia, hipermedia, acción comunicativa, representación del conocimiento, educación virtual, modalidad asincrona, ciclo de vida tecnológico.

\section{Presentación del grupo}

La informática educativa dentro de la Universidad Autónoma de Manizales (UAM) ha sido pensada desde la creación de la facultad de Ingeniería de Sistemas. Es así como desde diversos cursos del currículo, se han realizado actividades conducentes a reflexionar en torno al área.

Al interior de la Facultad, en el curso de Impacto Social de la Computadora, se planteó en el año 1994 trabajar el tema "Los efectos de la informática en la educación", lo que produjo como resultado la realización de un foro en Informática Educativa en noviembre del mismo año, en alianza con la especialización de informática educativa de otra universidad de la ciudad, en el que participaron como ponentes los coordinadores de la Red Iberoamericana de Informática Educativa, capítulo Colombia, y el decano de la facultad de Ingeniería de Sistemas de la Universidad Autónoma de Manizales. Este evento permitió hacer un primer acercamiento al estado del arte de la Informática Educativa en la región del Eje Cafetero.

En 1996, la facultad de Ingeniería de Sistemas se convierte en miembro activo de RIBIE-COL, se consolida el grupo de Informática Educativa (1-E) de la facultad y organiza un segundo foro regional en las instalaciones de la UAM; así mismo, inserta la Informática Educativa en el plan de estudios de la facultad como un área de énfasis opcional en la cual los estudiantes y profesores desarrollan trabajos de grado en software educativo, en algunos casos en coordinación con grupos de trabajo de la región, como el Comité de Cafeteros de Caldas.

En este mismo año y como una actividad paralela e independiente al grupo, algunos docentes de la universidad realizaron la especialización en Investigación y docencia universitaria, que dio origen a trabajos en el área de Informática educativa, tal es el caso de la propuesta: Modelo educativo: Universidad Multimodal. Parte de este trabajo fue implementado, en el mediano plazo por uno de los gestores de la propuesta, quien crea lo que hoy es el Sistema de Aprendizaje Interactivo Virtual (SAIV), encargado de programas en modalidad virtual para educación superior, empresas y otras poblaciones objetivo.

En el año 1997, La Facultad de Ingeniería de Sistemas solicita a la dirección de RIBIECOL, la sede y organización del IV congreso Colombiano de Informática Educativa, el cual fue realizado en Manizales en el año 1998, logrando un cubrimiento Nacional e Internacional, lo que permite consolidar aún mas la participación del grupo de informática educativa de la Facultad de Ingeniería de Sistemas en la red.

En 1999 integrantes del grupo y otros profesores de la universidad, inician estudios de maestría en Tecnología Educativa en el Instituto Tecnológico de Estudios Superiores de Monterrey (ITESM), fortaleciendo tanto al grupo de Informática Educativa, como al SAIV. Estos dos grupos de trabajo de la UAM, de manera permanente, se fortalecen en lo 
académico, metodológico y tecnológico, a pesar de su operatividad como unidades independientes.

En el año 2000, el grupo de informática educativa acompaña el proceso de transferencia tecnológica de Ludomática a instituciones y escuelas de los municipios de San José, Neira y Manizales apoyados y acompañados por el SENA, contribuyendo con ello al cumplimiento de uno de las metas del proyecto Ludomática del Laboratorio de Investigación y desarrollo en Informática Educativa de la Universidad de los Andes y la Fundación Rafael Pombo. Este proceso permitió la proyección del grupo de informática educativa en la zona rural de la región, su fortalecimiento académico a través de la experiencia vivida y el desarrollo de materiales educativos computarizados que incorporan los principios de Ludomática en trabajos de pregrado.

Durante estos años, el grupo de la Facultad de Ingeniería de Sistemas, se ha extendido a la comunidad a través de proyectos piloto de capacitación, como lo fue: El uso de juegos de computador para niños con deficiencia auditiva, servicios en análisis de necesidades educativas para diferentes instituciones de la ciudad y elaboración de propuestas y proyectos de investigación en el área. Además, se ha continuado con el desarrollo de proyectos de grado en informática educativa con participación de alumnos de pregrado vinculados al grupo, acompañados por los profesores de éste, permitiendo un mayor aporte a las necesidades de la región.

Actualmente las actividades del grupo se orientan hacia la fundamentación teórica y a la institucionalización de línea de investigación en informática educativa dentro de la UAM. Dicha fundamentación del grupo se traduce en su misión, visión y objetivos, que se presentan a continuación.

\section{Misión}

El grupo de informática educativa de la Universidad Autónoma de Manizales, trabaja en el desarrollo y mejoramiento de procesos educativos apoyados por tecnologías de información y de comunicaciones; con el fin de mejorar la cobertura y calidad de la educación en todos sus niveles en Colombia.

\section{Visión}

Para el año 2005 seremos una comunidad académica sólida, reconocida a nivel nacional por nuestra participación en proyectos de mejoramiento educativo.

\section{Objetivo general}

Desarrollar propuestas de investigación, desarrollo y formación con pertinencia social, en informática educativa a través de la realización de trabajos que permitan apoyar procesos educativos al interior y exterior de la Universidad en todos los niveles de educación.

\section{Objetivos específicos}

- Desarrollar proyectos de investigación, relacionados con el uso de tecnología en los procesos de enseñanza aprendizaje. 
- Asesorar en procesos de educación que requieran del uso de medios tecnológicos con base en el análisis de necesidades educativas.

- Capacitar a la comunidad en I.E. mediante actividades del área, tales como congresos, foros, cursos y publicaciones.

- Desarrollar software educativo y evaluar el existente de acuerdo a las necesidades educativas detectadas en el medio.

- Fortalecer los nexos con las comunidades académicas nacionales e internacionales por medio de actividades conjuntas.

- Proponer nuevos modelos pedagógicos que involucren el uso de la informática en la educación.

- Desarrollar proyectos en educación desescolarizada utilizando tecnología de telecomunicaciones.

\section{Avances teóricos del grupo}

La lectura de diferentes autores y la experiencia en el tema, han permitido al grupo concebir la informática educativa como una disciplina que estudia el uso eficiente de herramientas tecnológicas en los procesos de enseñanza y aprendizaje, apoyados por teorías educativas y pedagógicas, que le permitan a los partícipes, aprehender, compartir y generar conocimiento en torno a la ciencia, el arte y la técnica.

Ello significa, que esta disciplina no se centra únicamente en el uso del computador como herramienta educativa, sino que contempla el uso de otros productos tecnológicos que puedan agregar valor a situaciones de aprendizaje y de enseñanza, de acuerdo con los fines establecidos.

El grupo de informática educativa de la UAM, al originarse en la Facultad de Ingeniería de Sistemas, ha pensado sobre los supuestos teóricos, metodológicos y procedimentales que sustentan estas dos disciplinas, encontrando que ambas aprovechan el potencial de las nuevas tecnologías de información y comunicación existentes para desarrollar nuevos métodos que incrementan el conocimiento y se complementan con otras áreas del saber, a las cuales les agrega el componente reflexivo en su apropiación y uso

Teniendo en cuenta este último ingrediente, el reflexivo, los trabajos emprendidos por los integrantes del grupo en los últimos dos años han permitido diferentes estudios en torno al papel de las nuevas tecnologías de información y comunicación (NTIC) en la educación superior, particularmente en torno al tema de la multimedia en relación con los procesos de conocimiento.

Este análisis puede verse en (Quiceno, 2001), en donde la autora de esta propuesta interpreta las teorías de diferentes expertos en el tema como Jacquinot, Morín, Hadj y Delouis frente al tema del uso de tecnologías en educación.

Con base en dichos autores se puede concebir la información, como un elemento que surge de la dinámica propia del conocimiento, relación sujeto-objeto, el cual juega un papel importante en la permanencia del individuo dentro de una colectividad, o de la colectividad dentro del mundo. 
Con respecto a las nuevas tecnologías de información y comunicación (NTIC), éstas son productos que surgen en la última de las fases del sistema tecnológico: La de aplicación; Las fases que le anteceden son: la de los conceptos científicos, y la de los procedimientos técnicos (Hadj, 1990, p. 30), lo cual permite afirmar que un producto tecnológico guarda tras de sí la investigación científica de la cual emerge y de las disciplinas científicas y métodos que permiten su desarrollo, hasta legarlo a materializar en un objeto tangible o intangible, puesto por el hombre al servicio del hombre.

Es así como la evolución de la informática y las telecomunicaciones hicieron posible la aparición de las herramientas multimedia utilizando el computador como medio que las reúne. La multimedia nace como analogía de la forma natural como el hombre íntegra os datos de los objetos que percibe, ayudado por los órganos de los sentidos, que le permiten captar de múltiples maneras la información sobre el objeto. (Delouis 1997, pp. 57-60) denomina a esto último multimecha natural, y a lo primero multimedia artificial.

La multimedia artificial forma parte de otro producto tecnológico mayor denominado hipe media, cuyo origen está en el hipertexto. Éste aparece en el ámbito tecnológico como consecuencia de otra analogía con el aprendizaje natural, en donde el sujeto posee un conjunto de conocimientos en su memoria, los asocia de acuerdo a lo que ellos significan o a las imágenes que le evoca, formando un todo interrelacionado.

Desde una perspectiva cognitiva, se puede decir que la hipermedia es una forma de representar el conocimiento humano haciéndolo evidente a otros. La hipermedia (tecnológica) está compuesta por puntos de enlace que pueden contener diversas maneras de presentar la información, estos nodos pueden ser multimedia, lo cual permiten formar un sistema. Lo más interesante de estos sistemas hipermedia es que, al surgir de la emulación como los humanos procesamos la información, reflejan de manera directa la concepción de la representación de la información del individuo que las crea. El autor hipermedia o multimedia, está colocando, como pro-dueto tecnológico, su manera de ver el mundo, está haciendo público su entendimiento de éste, por ello, las representaciones multimedia e hipermedia son diferentes para cada tema que se quiera difundir, puesto que la representación es el reflejo de una interpretación individual: la de su autor.

Es interesante además saber que en esta representación, el autor refleja el presupuesto con relación al usuario; el autor hace con antelación el juicio de cómo aprende su usuario, razón por la cual dirige los enlaces, presenta los contenidos y los íntegra "de una cierta manera" es decir, el autor proyecta a través de múltiples medios su conocimiento y el de los demás a través de una representación que parte de lo mental para convertirlo en un producto tecnológico (Jacquinot, 2000).

Si al crear o utilizar las NTIC en la educación se quiere generar aprendizaje, se debe suponer que este medio tecnológico genera efectos similares a los que se dan en la interacción con el medio real, o en la multimedia natural; puesto que aquí, a través del medio tecnológico, llámese multimedia, hipermedia, se desarrollan maneras de pensar acerca del mundo que pueden ser reunidas en un solo centro: el software o el hardware, para ser comunicadas a un individuo (sujeto cognoscente).

Con base en los planteamientos anteriores surgen preguntas como ¿qué nuevos aportes hacen estas tecnologías?, ¿por qué son atractivas para los procesos de aprendizaje? La respuesta puede darse desde el sentido común: estas NTCl pueden generar conocimiento de realidades no inmediatas, incrementando jas posibilidades que 
puede ofrecer el medio natural, sin arriesgarlo. También puede suponerse que el aprendizaje que provoca las NTIC es mas integrado porque puede conjugar todos los sentidos por igual (con sistemas multimedia se puede hacer), sin embargo algunos estudios también han demostrado que pese a ventajas como las enunciadas, se han detectado productos y procedimientos, que hacen menos fácil y llamativo los procesos de aprendizaje, haciendo mal uso del potencial de las NTIC.

Este tipo de cuestionamientos generados desde lo teórico, son hoy motivo de investigación por parte de los integrantes del grupo de I-E de la UAM, quienes han originado propuestas de trabajo de campo que permitirán dilucidar sobre como se representa el conocimiento a través de herramientas hipermedia y cuál es su interpretación y efectos en quienes se benefician de ellas, para integrar en su diseño el buen uso de las mismas.

\section{Avances en lo metodológico}

En lo metodológico, el análisis realizado se concentra en la caracterización de los procesos de enseñanza en los cursos virtuales de la UAM, utilizando como población objetivo los estudiantes y docentes de la asignatura Espíritu Empresarial del área administrativa del macrocurrículo institucional (Rivero, 2001).

Este trabajo determina las estrategias de enseñanza utilizadas por el profesor que orienta el curso virtual, en modalidad asíncrona, y describe los procesos de interacción entre el profesor y los estudiantes y los estudiantes entre si en relación con los contenidos; además de la caracterización de los medios tecnológicos utilizados para llevar a cabo el proceso de enseñanza.

El análisis de la interacción social que se presenta en este trabajo de investigación, tomando como estudio de caso la asignatura Espíritu Empresarial en modalidad virtual, se fundamenta en lo propuesto por Habermas (1988, p. 122) en su discusión acerca de la teoría de la acción comunicativa, la cual se toma para el análisis, como el eje articulador. La interacción como actividad social puede ser entendida según el autor, como aquella en la que participan por lo menos dos sujetos capaces del lenguaje y la acción, quienes entablan una relación interpersonal en la búsqueda de negociación frente a un asunto en particular, lo cual en este caso, se hace explícito a través del uso de medios tecnológicos para la comunicación utilizada en el proceso de enseñanza y la estrategia utilizada por el profesor para desarrollar el curso y evaluarlo.

Una estrategia que permite la comunicación bidireccional asincrónica son los foros de discusión y el correo electrónico utilizados para compartir temáticas particulares del curso, propuestas por el profesor, que inciden en la construcción de aprendizajes en los estudiantes. Esta estrategia en sí misma es concebida por los actores del proceso educativo, como una posibilidad de enriquecimiento mutuo entre los estudiantes y el profesor, quienes la entienden como una oportunidad de expresión sin el temor que causa la comunicación cara a cara propia de los cursos presenciales.

En la práctica la investigación reportó que las ventajas mencionadas por los actores, se puede ver limitadas por factores contingentes, como los son el tiempo de dedicación, el cual debe ser compartido con asignaturas presenciales y la disponibilidad de los medios, los cuales se ven concentrados en sitios específicos de la institución, lo que imita su acceso por los horarios preestablecidos impidiendo la libertad de ejecutar una acción comunicativa en el momento en que se desee y se requiera. 
Además es necesario tener en cuenta que el conocimiento puesto en común a través de los medios implica un buen uso de éstos, el saber leer, reflexionar, sintetizar y construir una respuesta que refleje las nuevas estructuras conceptuales ocurridas en el individuo que comunica, atendiendo a las reglas preestablecidas en toda comunicación.

Algunos de los resultados mencionados llevan a recomendar en lo metodológico que quienes planean los cursos virtuales en las facultades de modalidad presencial, también reconozcan las implicaciones que lleva el abordaje de un curso en esta modalidad educativa en todas sus fases (concepción, diseño, implementación, distribución), de tal manera que se identifique antes de su distribución, el valor agregado de éste frente a la modalidad presencial.

Con respecto a las estrategias de enseñanza la investigación en referencia retorna la visión de Monereo (1990, p. 6) frente al tema, quien sostiene que cuando el profesor busca el aprendizaje de sus estudiantes, no sólo está instruyendo sobre el qué de la materia en cuestión (conceptos, procedimientos, principios/valores), sino que simultáneamente está mostrando el cómo tratar y presentar/representar este contenido para ser aprendido. La visión de este autor pone de manifiesto que la estrategia que utiliza el profesor en su proceso de enseñanza, contempla tanto los objetivos e intencionalidades del curso (Qué), como de la representación que éste concibe del conocimiento a ser presentado (Cómo).

En referencia con el primer elemento señalado desde esta fuente teórica, el qué, el análisis de la información recolectada, deja ver que el profesor tiene concebida una estrategia metodológica que contempla el qué del curso y lo que se espera de los estudiantes en el mismo.

En cuanto al segundo elemento puesto de manifiesto en la aproximación teórica realizada por Monereo (1990, p. 6), el cómo, se puede ver que el autor considera que los profesores hacen una representación de los contenidos a ser presentados a los estudiantes para que ellos los apropien. Es decir, el autor concibe que los conocimientos presentados por el profesor a los estudiantes, no corresponden con los objetos de conocimiento que existen en la realidad; puesto que los profesores toman éstos y les imprimen sus emociones, saberes y concepciones transformándolos en objetos de enseñanza (un saber mediado por el profesor).

Frente a este elemento (el cómo), la investigación deja ver que el asunto sobre cómo representa el conocimiento el profesor en el curso virtual analizado, no ha sido completamente objeto de estudio por parte de éste, a pesar de haber participado en procesos de educación en modalidad virtual donde cómo representar el conocimiento juega un papel primordial, que siempre se pospone por dar cumplimiento a las actividades propias del curso.

Como se mencionó desde la perspectiva teórica el grupo de Informática Educativa de la UAM, convierte esta carencia en una necesidad para ser investigada.

Por otro lado, el grupo se ha preocupado por plantear estrategias de enseñanza de la programación en modalidad a distancia apoyadas por tecnología informática; por esta razón actualmente se adelanta un trabajo de grado de la Maestría en Tecnología Educativa que apunta a solucionar esta inquietud, realizado por uno de los miembros del grupo. 
La computación es un disciplina que tiene como objetivo modelar y representar en un computador sistemas de conceptos u objetos con el fin de resolver problemas, realizar predicciones mediante la simulación de sus interacciones o efectuar cálculos y raciocinios con ellos; por consiguiente, el proceso de aprender a programar es complejo, la computación es una disciplina que obliga al estudiante a desarrollar jerarquías conceptuales que son mucho más profundas que en otras áreas del saber (Dijkstra,1989). Por lo tanto al enseñar computación se deben desarrollar habilidades en pensamiento lógico que permitan al estudiante realizar las abstracciones necesarias con el fin de identificar problemas y resolverlos utilizando herramientas computacionales, lo cual en Educación a Distancia puede ser aún mas complejo.

Los modelos educativos de hoy día requieren hacer del aprendizaje distribuido apoyado por tecnología una manera de actuar en los proceso de enseñanza-aprendizaje. Es decir buscar nuevas estrategias de enseñanza que lleven a los estudiantes a tener diversos caminos en su proceso de aprendizaje. Un ambiente de aprendizaje distribuido es un enfoque educativo centrado en el estudiante, el cual integra diversas tecnologías que permiten realizar actividades diversas independientes del tiempo o espacio donde actúe el alumno, pero también permite realizar comunicaciones de manera sincrónica pero apoyadas por las nuevas tecnologías de información y comunicaciones (Bates, 1999).

\section{Avances en lo tecnológico}

El desarrollo de software educativo como una de las actividades del grupo, se ha materializado por medio de trabajos de pregrado que han permitido establecer la pertinencia de los métodos de desarrollo de software propuestos desde la ingeniería, además de hacer posible su conjunción con propuestas surgidas desde el análisis de necesidades educativas que permiten trabajos interdisciplinarios con otros grupos de investigación, siendo esto un aporte importante para el contexto regional y social. Es así como se ha implementado el aporte de las NTIC a áreas como la psiquiatría, lenguaje, las finanzas; además de desarrollos que involucran la cultura regional.

\section{Bibliografía}

Bates, T. (1999) La tecnología en la enseñanza abierta y la educación a distancia. México, Trillas.

Delouis, S. (1997) Hypermedia etprocessus d' apprentissa ge, Dans Representa tions Mentales et Nouvelles Technologies Educativos. Actos Des Journees Des 17 et 18 Décember, INSA De Lyon et Université Lyon 2, p. 52.

Dijkstra, E. (1989) "A debate en teaching computing science", in Communications of ACM. USA, Volumen 32, No. 12.

Galvis, A. (1997) Ingeniería de software educativo. Bogotá, Ediciones Uniandes.

Habermas, J. (1988) Teoría de la acción comunicativa. Madrid, Taurus.

Hadj, S. (1990) Gestión de la tecnología. La empresa ante la mutación tecnológica. Addison-wesley, Iberoamericana, SA., p. 30. 
Jacquinot, G. (1998) Cinéma et Derniéres Technologies. Sous Directionn de F. Beau, P., Dubois, G., Leblanc, Paris, NA/De Boeck, coll Ars et Cinéma.

Monereo, C. (1990) Las estrategias de aprendizaje en la educación formal: enseñar a pensar y sobre el pensar, infancia y aprendizaje, 50, 3-25.

Monereo, C. (1998) Estrategias de enseñanza y aprendizaje. Barcelona, Graó.

Moore, M., Kearsley, G. (1996) Distance Education, A system view. USA, Wadskworth Publishing company.

Quiceno, H. (2001) Efectos de las NTIC en los procesos de aprendizaje en la educación superior (propuesta). Colombia.

Rivero, M. (2001) Los procesos de enseñanza en los cursos virtuales de la UAM: Curso espíritu empresarial del área administrativa del macrocurrículo institucional. Colombia.

Rueda, F. (1997) "Consideraciones para la actualización y la modernización del currículo de ingeniería de sistemas" en Revista Sistemas, Colombia, Asociación Colombiana de Ingenieros de Sistemas.

SAIV, (2001) "Universidad Autónoma de Manizales", en: www,autonoma,edu,cp/ travesias/cursos/modulos/virtual/concopto/html 